\title{
Intelligent Detector Design
}

\author{
Norman Graf $^{1}$, Steve Magill ${ }^{2}$, Steve Kuhlmann ${ }^{2}$, Ron Cassell ${ }^{1}$, Tony \\ Johnson $^{1}$, Jeremy McCormick ${ }^{1}$ \\ ${ }^{1}$ Stanford Linear Accelerator Laboratory, Menlo Park, CA 94025 \\ ${ }^{2}$ Argonne National Laboratory, Argonne, IL 60439
}

\begin{abstract}
At a future e+e- linear collider, precision measurements of jets will be required in order to understand physics at and beyond the electroweak scale. Calorimetry will be used with other detectors in an optimal way to reconstruct particle 4-vectors with unprecedented precision. This Particle Flow Algorithm (PFA) approach is seen as the best way to achieve particle mass resolutions from dijet measurements in the range of $\sim 30 \% / \sqrt{E}$, resulting in innovative methods for choosing the calorimeter technology and optimizing the detector design.
\end{abstract}

Keywords: Calorimeter, Particle Flow, Linear Collider

PACS: 07.20.Fw, 29.40.Vj, 07.05.Kf

\section{PARTICLE RECONSTRUCTION GOALS}

At the future $\mathrm{e}^{+} \mathrm{e}^{-}$international linear collider (ILC), precision measurements of jets of particles will be required in order to fully explore and understand the (possibly subtle) effects of the new physics expected at and beyond the electroweak mass scale. A precision measurement of the 4-vector associated with a quark or gluon initiating a jet is best done by fully reconstructing the individual particles produced in the $\mathrm{e}^{+} \mathrm{e}^{-}$ annihilation process. This requires the development of so-called Particle Flow Algorithms (PFAs) which, in combination with an optimized detector, can reconstruct these particles. It is the calorimeter, which must be optimized for the PFA approach, that is the key element which makes possible a precise measurement of both energy and momentum of the particles. For calorimetry, this means that high segmentation and granularity leading to individual particle shower reconstruction is preferred over energy compensation; the loss of good calorimetric energy resolution is more than offset by the gain achieved using the charged particle momenta from tracking.

In this report, the effect of PFA development on calorimeter design for the ILC will be discussed along with a description and results of a particular implementation of the PFA approach for different calorimeter models.

\section{CALORIMETRY REQUIREMENTS FOR PARTICLE FLOW}

In the PFA approach to individual particle reconstruction, the goal is to use the best measure of a particular particle's properties - this means that all charged particle momenta are taken from tracking measurements, photon energy is detected and

Contributed to 12th International Conference on Calorimetry in High Energy Physics (CALOR 06), 06/05/2006--6/9/2006, Chicago, Illinois

Work supported in part by the US Department of Energy contract DE-AC02-76SF00515 
measured in the electromagnetic calorimeter (ECAL), and neutral hadrons are measured in the ECAL and the hadron calorimeter (HCAL). For this to work, the most important part of the PFA is separation of charged hadron showers from neutral hadron showers in the calorimeter. Therefore, it is 3-D shower reconstruction performance in the calorimeter that drives the calorimeter technology choice and design. A dense absorber is required, especially at the beginning of the calorimeter, in order to best separate the shower starting points of electromagnetic particles from hadrons. As the ratio of hadronic interaction length to radiation length $\left(\lambda_{\mathrm{I}} / X_{0}\right)$ grows, the starting points of hadron showers are pushed farther into the calorimeter on average, thus providing good longitudinal separation between e.g., photons and neutrons. In a dense calorimeter, the Molière radius ( $\left.\mathrm{r}_{\mathrm{M}}\right)$ is small, and to obtain good transverse shower separation, cells should be of the order of $r_{M}$ in lateral size. A typical choice for the ECAL at the ILC is a 30 layer sandwich of tungsten absorber ( 2/3 $X_{0}$ per layer) with silicon readout cells of size $\sim 3.5 \mathrm{~mm} \times 3.5 \mathrm{~mm}$ [1].

In the HCAL, complete separation of charged and neutral hadron showers requires that high granularity and segmentation be continued. The size of an HCAL cell should be optimized for single particle occupancy of any individual cell, thus allowing high purity shower reconstruction. For most particles, digital readout of the HCAL cells is more than adequate for both shower reconstruction and precise energy determination. Figure 1 shows a comparison of traditional analog calorimetry with a digital method for a $5 \mathrm{GeV} \pi^{+}$. The analog measurement displays Landau broadening of the distribution due to the characteristic minimum ionizing particle (MIP) response and path length effects - both of these are absent in the digital measurement, resulting in improved resolution.
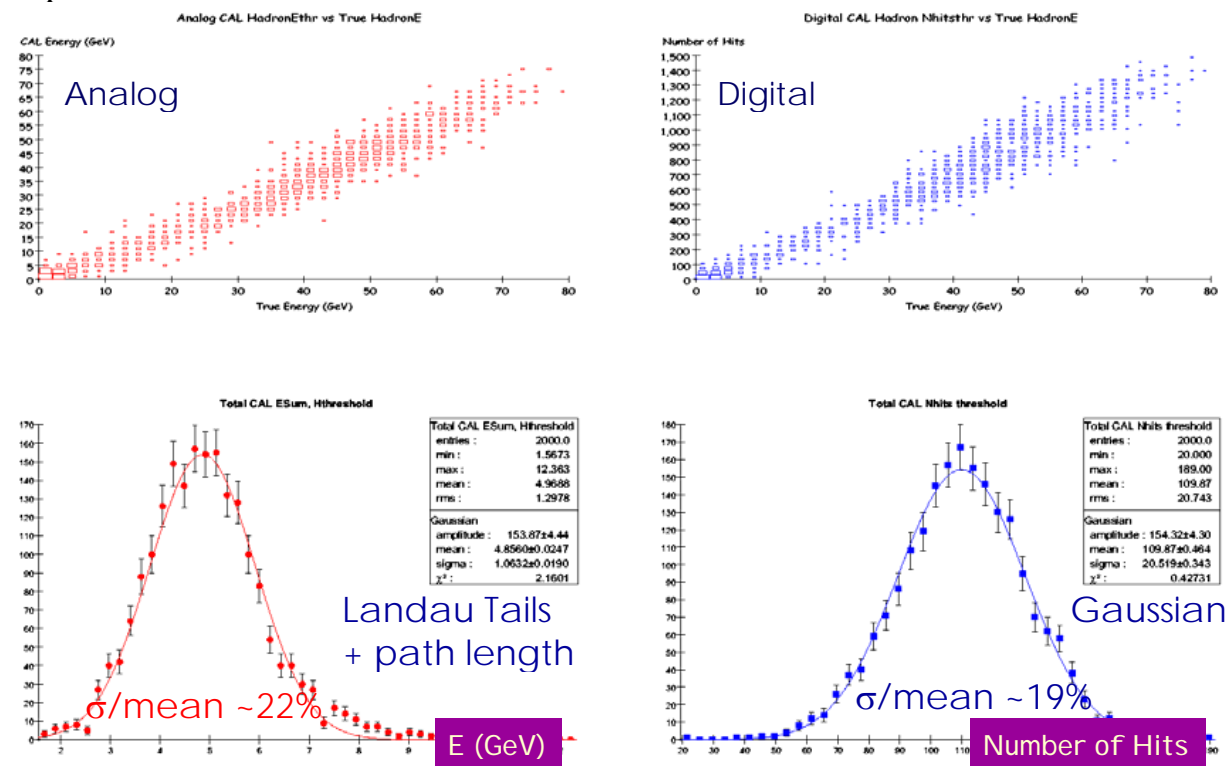

FIGURE 1. (left) Analog calorimeter linearity of response (top) and energy resolution (bottom) compared to (right) digital calorimeter linearity of response (top) and energy resolution (bottom). 
Active media choices for the sampling calorimeters under investigation are traditional (but small) scintillator tiles with SiPM readout [2] and technologies employing gas (RPC [3] and GEM [4]). Scintillator is a good neutron detector due to the possibility of detecting elastically scattered protons from incident neutrons as opposed to the gaseous detectors which are somewhat insensitive to neutrals. However, the smaller, better-separated neutral showers in a gaseous detector may enhance the performance of the PFA, more than offsetting the poor neutral energy resolution response of gas. Since the whole calorimeter should be inside a strong solenoidal magnetic field, use of a dense absorber here can keep the magnet bore (and therefore cost) as small as possible.

The PFA approach is used to optimize both jet reconstruction and to optimize the calorimeter design for particle reconstruction. This requires a flexible, fast, full detector simulation with an investigation of many different detector models and options, including variations of detector parameters such as inner detector radius. A complete simulation package has been developed with this in mind [5], and has been used to "build" more than a dozen detector models, each with many single parameter variations. Physics processes at $500 \mathrm{GeV}$ center-of-mass energies and single particle simulations are all included with each detector design variation. Also, for initial development of PFAs, $\mathrm{e}^{+} \mathrm{e}^{-} \rightarrow$ qq events at the $\mathrm{Z}$ mass are generated; these are very useful since PFA performance can be evaluated with simple energy sums and no jet algorithm interference. These analyses also provide a point of reference for the LEP and SLC detectors.

Also, since separation of showers in the calorimeter is crucial to PFA performance, the simulation of hadronic showers is of critical importance. Timely test-beam results are needed to validate the hadron shower simulations and verify the feasibility of the PFA approach to detector optimization.

\section{INDIVIDUAL PARTICLE RECONSTRUCTION STRATEGY}

The approximate energy content of all jets in $\mathrm{e}^{+} \mathrm{e}^{-}$annihilation is: $60 \%$ charged hadrons, $25 \%$ photons, $10 \%$ neutral hadrons, and 5\% missing energy (neutrinos) as shown in Figure 2 (left). Of course, there are large fluctuations in these quantities per event. In Figure 2 (right), the energy distributions of the particles are shown. 


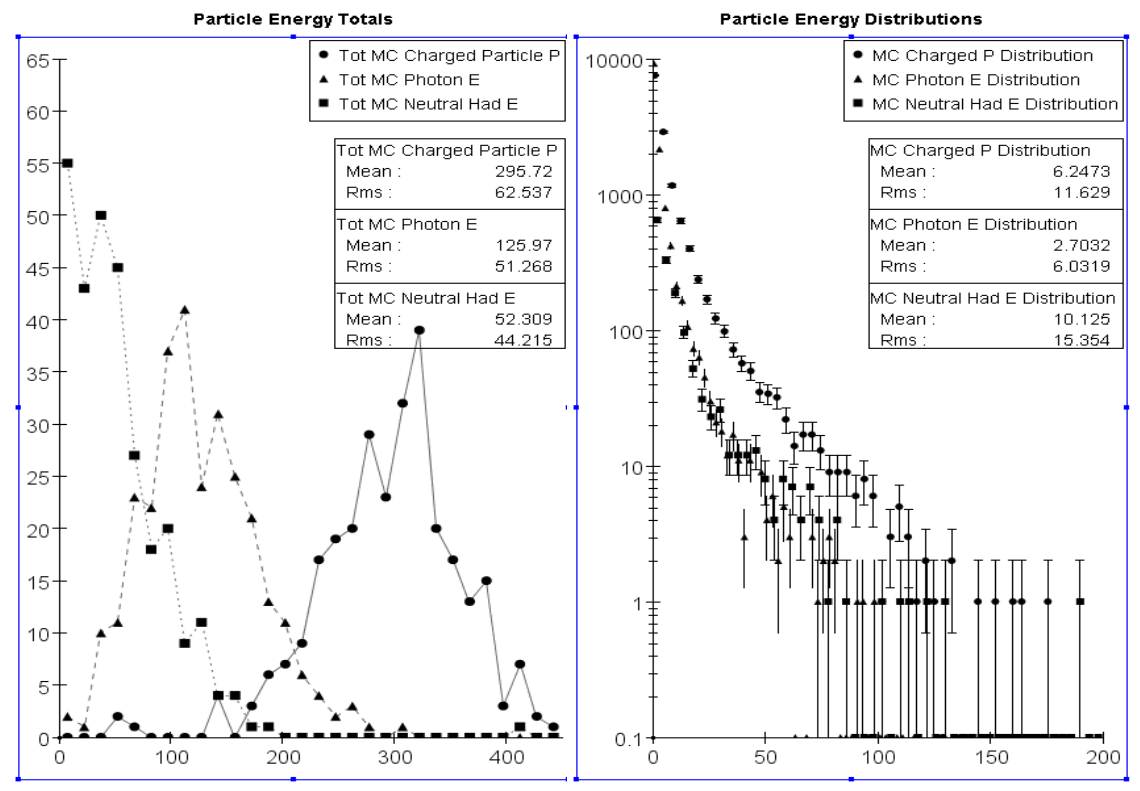

FIGURE 2. (left) Total particle energies and (right) particle energy distributions for typical events in $\mathrm{e}^{+} \mathrm{e}^{-}$collisions at $500 \mathrm{GeV}$ CM.

Starting with the largest energy fraction, tracks from charged particles are extrapolated into the calorimeter, associating MIP hits until the interaction layer is found - simply characterized as a layer with either no hits or many hits in a small window around the extrapolated track. The calorimeter hits in the resulting MIP "clusters" associated with tracks are removed from further evaluation. Typically, this step results in single-cell roads removed primarily from the ECAL. Next, cells in the ECAL are clustered with a suitable cluster algorithm (cone clusterer, directed tree [6], nearest-neighbor, etc.). All clusters are then tested with an H-Matrix algorithm which forms a chi-squared probability for each cluster based on longitudinal energy correlations which has been trained on real photon clusters. Clusters which pass this test are designated as photons and saved for input to the jet algorithm. The calorimeter hits from photon clusters are then removed from further evaluation. In principle, what is left in the calorimeter now are hit cells from neutral hadrons and the hits of charged hadrons without their MIP tails in both the ECAL and HCAL. These hits are now clustered with a nearest-neighbor cluster algorithm. Continuing the extrapolation of tracks from the end of their respective MIP clusters, the nearestneighbor cluster fragments are associated with the track if within a small iterated cone. The cone size is gradually increased, adding the energy of cluster fragments until a tuned value of E/p, the ratio of summed calorimeter cluster energy divided by the track's momentum, is reached, typically the value is $\sim 1-2 \sigma_{\text {had. }}$. All hits from clusters associated with tracks are removed from further evaluation. What is now left is due to neutral hadrons plus, of course, any mistakes made in all of the previous algorithms. Additional clustering can be done here, as well as other evaluations of the hits to 
define the neutral hadron clusters, which are then saved to be evaluated in a jet algorithm along with the photons and tracks. This completes the PFA. A picture illustrating the performance of this algorithm is shown in Figure 3.
$6.6 \mathrm{GeV} \gamma$
$1.9 \mathrm{GeV} \gamma$
Mip trace/lL
$1.6 \mathrm{GeV} \gamma$
$3.2 \mathrm{GeV} \gamma$
$0.1 \mathrm{GeV} \gamma$
$0.9 \mathrm{GeV} \gamma$
$0.2 \mathrm{GeV} \gamma$
$0.3 \mathrm{GeV} \gamma$
$0.7 \mathrm{GeV} \gamma$
$4.2 \mathrm{GeV} \mathrm{K+}$
$4.9 \mathrm{GeV} p$
$6.9 \mathrm{GeV} \pi-$
$3.2 \mathrm{GeV} \pi-$
$8.3 \mathrm{GeV} \overline{\mathrm{n}}$
$2.5 \mathrm{GeV} \mathrm{K}_{\mathrm{L}}{ }^{0}$

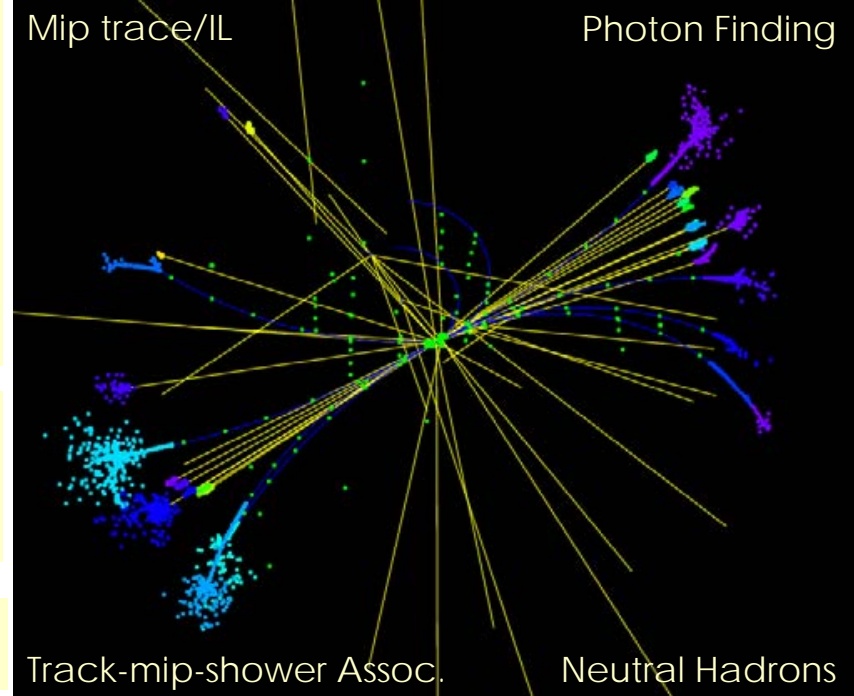
$1.9 \mathrm{GeV} \gamma$
3.7 GeV $\gamma$
3.0 GeV $\gamma$
$5.5 \mathrm{GeV} \gamma$
$1.0 \mathrm{GeV} \gamma$
$2.4 \mathrm{GeV} \gamma$
$1.3 \mathrm{GeV} \gamma$
$0.8 \mathrm{GeV} \gamma$
$3.3 \mathrm{GeV} \gamma$
$1.5 \mathrm{GeV} \gamma$
$1.9 \mathrm{GeV} \pi-$
$2.4 \mathrm{GeV} \pi-$
$4.0 \mathrm{GeV} \pi-$
$5.9 \mathrm{GeV} \pi+$
$1.5 \mathrm{GeV} \overline{\mathrm{n}}$
$2.8 \mathrm{GeV} n$

Overall Performance: PFA 33\%/ VE central fit

FIGURE 3. Illustration of PFA performance for an e+e- $\rightarrow \mathrm{Z} \rightarrow$ qq event at $91 \mathrm{GeV}$.

In this example, all particle showers have been correctly associated with the exception of a $0.1 \mathrm{GeV}$ photon - these photons rarely pass the minimum number of hits requirement for testing with the H-Matrix. A 2-gaussian fit of the response of this PFA at the Z-Pole yields a central peak fit result corresponding to $33 \% / \sqrt{\mathrm{E}}$. Also, for Z-Pole events, the "confusion" term - the contribution to the resolution due to algorithm mistakes - is smaller than the contribution from the neutral hadron measurement in the calorimeter.

\section{RESULTS FOR DIFFERENT DETECTOR MODELS}

Once the PFA performs at a level in which the calorimeter neutral hadron resolution term is the largest contribution to the total resolution, the algorithm can be used to test different detector configurations. It is assumed that the PFA approach will be sensitive to, among other things, the value of the solenoidal magnetic field and the radius of the calorimeter for a given calorimeter technology.

\section{Magnetic Field Variation}

The B-field was varied from its default value of 5 Tesla to 4 Tesla for the same detector design. In this detector, the ECAL was of W/Si sandwich construction, 
consisting of 30 layers of 2/3 $\mathrm{X}_{0}$ each with an inner radius of the barrel ECAL of 125 $\mathrm{cm}$. The HCAL was a Stainless Steel/RPC sandwich consisting of 34 layers of $0.12 \lambda_{\mathrm{I}}$ each. Figure 4 show the results of this comparison.
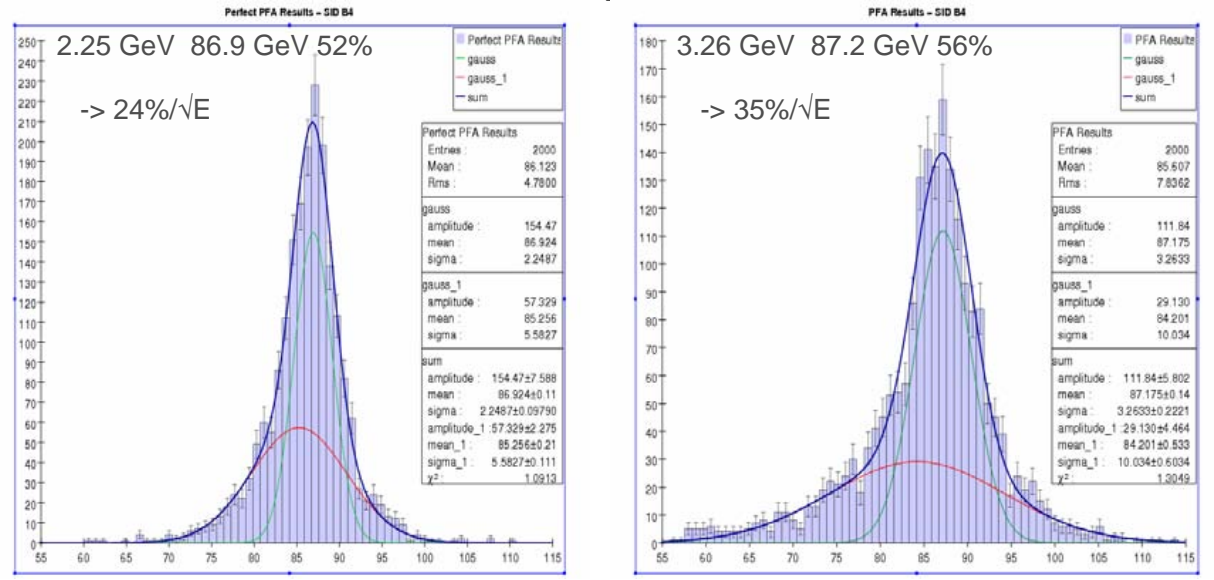

FIGURE 4. (left) PFA result for Z-Pole events in 5 Tesla field detector and (right) in a 4 Tesla detector.

The PFA distribution for the higher B-field model has better resolution than that for the lower field as expected. Not only is the resolution better, but as a consequence, the mistakes made in the PFA are smaller for the high B-field model than for the lower. As expected, increasing the B-field makes the PFA easier to perform and results in a better overall energy resolution.

\section{Calorimeter Radius Variation}

Another variation that should affect the PFA result is the distance from the interaction point (IP) to the calorimeter. A detector model was constructed with the inner radius of the barrel ECAL increased from its default value of $125 \mathrm{~cm}$ to $150 \mathrm{~cm}$. A further optimization was made in the HCAL, changing from a Stainless Steel/RPC HCAL to one made of W/Scintillator layers. Since tungsten is denser than stainless steel, the similar depth $\left(\sim 4 \lambda_{\mathrm{I}}\right)$ HCAL was $\sim 22 \mathrm{~cm}$ thinner than the default one, resulting in the radius of the solenoid coils to only be increased by 1 inch. So, this detector has moved the calorimeter showers farther from the IP without increasing the magnet bore and the cost of the solenoid. Figure 5 shows the results of the PFA comparison of these models. 

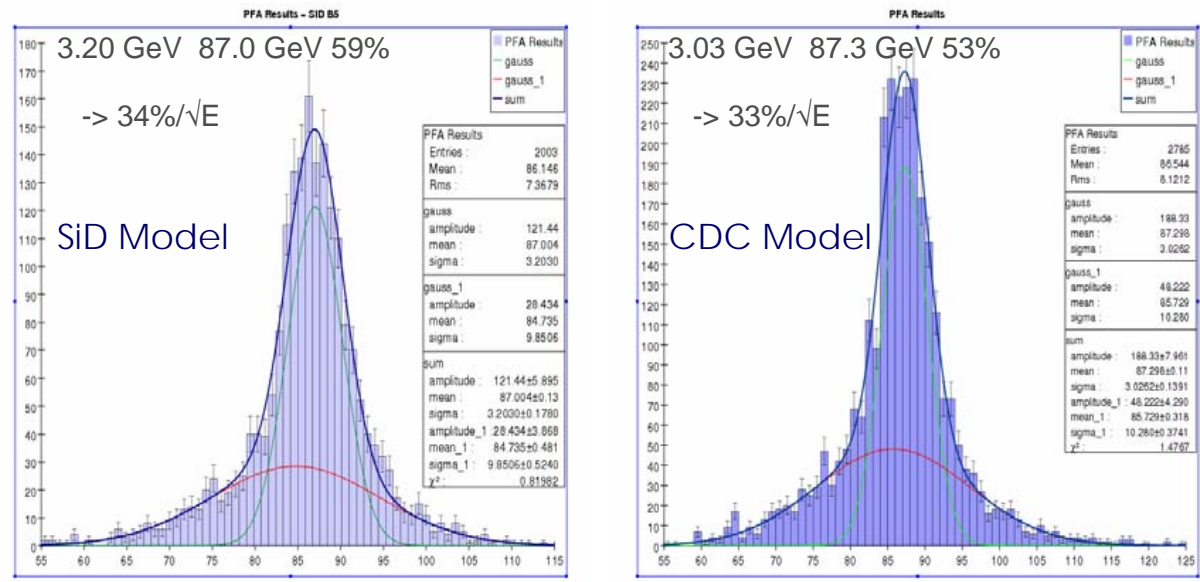

FIGURE 5. (left) PFA result for $125 \mathrm{~cm}$ radius ECAL and SS/RPC HCAL (SiD Model) compared to (right) the PFA result for $150 \mathrm{~cm}$ ECAL with W/Scintillator HCAL (CDC Model).

The results are similar for these detectors, with a slightly better result for the detector with larger ECAL radius, as expected. This illustrates how PFAs can be used to test different detector models, resulting in an optimized detector configuration which can then be prototyped and placed in a test beam to verify shower characteristics.

\section{PFA IMPLEMENTATION}

The PFA approach of reconstructing individual particles in an optimized detector lends itself to modular organization of the reconstruction algorithms. A PFA template has been developed to allow substitution of various clustering and analysis algorithms at each stage of particle reconstruction, thus resulting in faster optimization of a particular set of cluster and analysis codes. It also provides a way of testing individual reconstruction techniques as they are applied. This approach is seen as the best way to combine the efforts of the worldwide community working on a PFA approach to detector design and optimization.

\section{SUMMARY}

The PFA approach, combining full simulation with individual particle reconstruction, is a unique approach to detector - and in particular, calorimeter design. This is seen as the best way to optimize the detector configuration at the ILC for precise jet reconstruction. A particular implementation of cluster and analysis algorithms in a full PFA package has been developed and tested on fully-simulated $\mathrm{e}^{+} \mathrm{e}^{-}$data. The results are approaching the hoped-for goal of $\sim 30 \% / \sqrt{\mathrm{E}}$ with a nondominant "confusion" term. First tests of this algorithm on several calorimeter configurations have produced results illustrating how PFAs can be used to optimize the ILC detector. 


\section{ACKNOWLEDGMENTS}

This work is supported in part by the University of Chicago as Operator of Argonne National Laboratory under Contract No. W-31-109-ENG-38 and in part by Stanford University as Operator of the Stanford Linear Accelerator Center under Contract No. DE-AC03-76SF00515, both with the U.S. Department of Energy.

\section{REFERENCES}

1. N. Graf, Proceedings of the XII International Conference on Calorimetry in High Energy Physics (2006).

2. H-G. Moser, Proceedings of the XII International Conference on Calorimetry in High Energy Physics (2006);

E. Garutti, Proceedings of the XII International Conference on Calorimetry in High Energy Physics (2006).

3. J. Repond, Proceedings of the XII International Conference on Calorimetry in High Energy Physics (2006).

4. A. White, talk at XII International Conference on Calorimetry in High Energy Physics (2006).

5. N. Graf, Proceedings of the XII International Conference on Calorimetry in High Energy Physics (2006).

6. G. Lima, Proceedings of the XII International Conference on Calorimetry in High Energy Physics (2006). 